\title{
La Arqueoastronomía y la conservación del patrimonio cultural hondureño
}

Santos Vito Veliz

\section{Resumen}

Este trabajo trata de recoger todas las experiencias que se llevaron a cabo en estudios arqueoastronómicos, con respecto al Sol, en el Patio del Sol, Copán Ruinas. La idea es sacar de estas experiencias lo más relevante en cuanto a la conservación del patrimonio cultural de Honduras. Estas experiencias se realizaron en el campo, en los días de eventos especiales del Sol, visibles a simple vista. Esos días son los referentes a los equinoccios, a los pasos del sol por el cenit, al solsticio de verano y al solsticio de invierno. De antemano se seleccionaban los monumentos con los que se esperaba se alineara la salida o la puesta del sol por el horizonte y luego se iba temprano a las ruinas por la mañana o por la tarde, para comprobar los alineamientos. Fueron varios los alineamientos que se encontraron, tanto en la salida como en la puesta del sol. Los resultados más importantes fueron los alineamientos que se logró comprobar existían en Copán, con respecto al Sol, en lo que nosotros ahora llamamos el Patio del Sol. Tres resultados muy llamativos son los alineamientos entre graderías y las salidas o las puestas del sol. Dos de estos resultados son las direcciones de las graderías norte del Patio y la dirección de las graderías de la Estructura 223. La gradería noroeste alineada con la salida del sol en el solsticio de verano, la gradería noreste alineada con los equinoccios y la Estructura 223 alineada con la puesta del sol durante el paso del sol por el cenit. Llevamos estos resultados a los estudiantes y al público en general para dar a conocer nuestros hallazgos e invitar a la gente a cuidar estos restos de gran importancia para nuestro país.

Palabras claves: Arqueoastronomía, Arqueoastronomía Cultural, Conservación, Patrimonio Cultural, Identidad

Santos Vito Veliz, Departamento de Arqueoastronomía y Astronomía Cultural, Universidad Nacional Autónoma de Honduras 


\section{Introducción}

En la primera mitad del Siglo XVIII, algunos astrónomos comenzaron a identificar y a tomar en serio la relación directa entre algunos cuerpos celestes y ciertas obras humanas antiguas en el globo terráqueo. La creciente acumulación de datos al respecto llevó a estos investigadores al planteamiento de que el ser humano había tomado en consideración los astros para construir, ubicar y orientar ciertos monumentos. Aunque los arqueólogos no estaban totalmente de acuerdo. El hallazgo de estas relaciones se corrobora no con cualquier obra ni en cualquier día. Nuestros antepasados se valieron de aquellos días significativos en los movimientos anuales de los astros. Son estos los días cuando esos astros están en puntos horizontales fácilmente identificables para quienes observan cuidadosamente. En la actualidad, a ésos se les ha denominado días de eventos especiales. Con respecto al Sol, a esos días se les conoce como solsticios, equinoccios y pasos del sol por el cenit. A excepción del paso del sol por el cenit, estos eventos se experimentan por todo el planeta tierra, nada más que a la inversa en ambos hemisferios. El evento del paso del sol por el cenit es restringido porque éste sucede solamente en la banda tropical terrestre. Ésta es la banda este-oeste del globo terráqueo que se extiende 23.5 grados a cada lado del Ecuador terrestre, es decir, entre los trópicos: Trópico de Cáncer por el norte y Trópico de Capricornio por el sur del Ecuador. Dentro de esa banda tropical florecieron todas las más reconocidas civilizaciones de América Antigua: Chimú, Inca, Olmeca, Maya, Azteca y otras.

Alrededor de 1740 comenzó una serie de escritos sobre Stonehenge y luego sobre los templos de Egipto. En la década de 1960, una nueva generación de investigadores retomó la investigación arqueoastronómica en Inglaterra. Entre ellos estaban C. A. Newham quien en 1963 y el astrónomo Gerald S. Hawkins en 1963 y en 1964 hicieron publicaciones que revivieron el interés tanto en los alineamientos como en la controversia con los arqueólogos. Pero no fue sino hasta que Gerald S. Hawkins publicó en 1965 su libro sobre Stonehenge, que comenzó el diálogo constructivo con los arqueólogos. Comenzó entonces una serie de estudios de campo en diferentes partes del mundo, demostrando que el humano se había venido guiando por los astros para realizar obras arquitectónicas y artísticas.

En los Estados Unidos han sobresalido Aveni (2005) y Carlson (1999).

Ha habido también investigadores mexicanos quienes han dejado sus huellas en estos esfuerzos arqueoastronómicos: Broda (1982), Iwaniszewski (1982) y Maupomé (1982). 
En el caso de Honduras, el estudio más temprano que encontramos lo hizo Sylvanus Morley en la década de 1910 (1920), cuando encontró el alineamiento entre las Estelas 10 y 12. Le siguieron Aveni (1976) Anthony Aveni y Horst Hartung (1976), presentando otros alineamientos a finales de la década de los setentas. Hohmann y Vogrin (1982), arquitectos austríacos, en 1982 mencionan alineamientos en Copán. William Fash, Victoria y Harvey Bricker también tocan el tema de arqueoastronomía. En los ochentas, LeRoy Joesink Mandeville hace una breve referencia de alineamientos astronómicos en el sitio de Yarumela, Valle de Comayagua. Finalmente, son María Cristina Pineda de Carías, Vito Véliz y Ricardo Agurcia Fasquelle quienes publican sus trabajos en la primera década de este siglo, sobre sus hallazgos en Copán (Pineda de Carías, Véliz y Agurcia Fasquelle 2002, 2002a).Con estos esfuerzos, todos estos estudiosos han venido poniendo un granito de arena en el conocimiento del patrimonio cultural de la nación.

La UNESCO dice que el patrimonio cultural implica un conocimiento del tema y un respeto hacia el mismo, contribuyendo a diseminar el conocimiento y con obras para mantenerlo, preservarlo y conservarlo. No se ha podido hacer mucho con obras, pero sí con el conocimiento de nuestro legado cultural. Se ha desarrollado clases en la Universidad sobre Arqueoastronomía, Seminarios varios, charlas, Diplomados y mucho conocimiento por los medios de comunicación. Se considera éste un buen comienzo, dar a conocer al público lo que se ha encontrado, para que ellos mismos lo aprecien y lo sepan valorar y conservar. No hay mejor forma de impulsar la conservación de algo que darlo a conocer.

\section{Métodos y Técnicas}

En los trabajos realizados para esta investigación, hemos utilizado el trabajo conjunto. Se inició básicamente en el año 1999, entre la Profesora María Cristina Pineda de Carías, en aquel entonces Directora del Observatorio Astronómico Centroamericano de Suyapa (OACS) de la UNAH y el arqueólogo Ricardo Agurcia Fasquelle de la Asociación Copán. A partir del 2000, hubo varios años que se hacía hasta seis visitas a las Ruinas de Copán, para observar todos los eventos solares anuales. Me refiero a los equinoccios, pasos del sol por el cenit y solsticios. Cada uno de estos eventos sucede dos veces al año y se trataba de observar cada uno de ellos.

Por lo general, yo hacía arreglos telefónicos desde Tegucigalpa con el Representante del IHAH en Copán Ruinas, Profesor Oscar Cruz, con Ricardo Agurcia, 
con Seiichi Nakamura o con el Ing. Raúl Welchez, del Hotel Marina, para que me ayudaran a realizar las observaciones. Les pedía gente y materiales y ellos me lo proporcionaban. También solicitaba ayuda con walky-talkies y con binoculares. La oficina local de la ENEE también colaboraba con una escalera y mecates para poder subir a los árboles. Luego me movilizaba desde Tegucigalpa hacia Copán Ruinas unos cuatro o cinco días antes. El día del viaje, trataba de estar en las ruinas como a las 2:00 pm, platicar con la gente quien había prometido ayudar y luego platicar con los seleccionados para reunirnos al día siguiente.

El equipo estaba completo cuando contaba con una persona que anduviera con el walky-talky, cerca de donde estaban trabajando los hombres y tenía otros dos muchachos para movilizar y acarrear la escalera, juntar y picar las ramas. El equipo se completaba con dos muchachos más, quienes andaban en los árboles, cortando las ramas que se les indicaba. Desde el punto de observación, yo miraba hacia el este o el oeste e indicaba las ramas que había que cortar. Yo le decía al asistente, con el otro walky-talky, hacia donde tendrían que moverse los muchachos en el árbol y qué ramas cortar. El trabajo era muchas veces peligroso, especialmente por dos razones. Una debido a la altura de algunos árboles y al hecho de que no tenían ramas. Es normal que en un lugar, donde no hay mucho espacio, los árboles traten de crecer para alcanzar aunque sea un poco de la luz del sol. Otra razón por lo peligroso del trabajo, es el hecho de que se trataba de no hacer un abra muy ancha ni cortar muchas ramas. Entonces había que trabajar en situaciones difíciles, tratando de llegar hasta ramas muy pequeñas, donde uno no podía pararse muy bien.

Los muchachos hacían un muy buen trabajo, desde las 7:00 am hasta las 3:00 pm. Para el corte de las ramas se había llegado a un arreglo con el IHAH, de cortar lo mínimo posible. El corte de ramas tenía que ser el mínimo, no sólo para respetar y conservar la arboleda que por más de cien años ha crecido entre las ruinas, sino también para preservar las ruinas abajo. Aunque hayan sido la causa de destrucción de ruinas, estos árboles son, hoy día, el sustento y la conservación de estas ruinas. En Copán Ruinas se ha descubierto muchos casos en los cuales los árboles, con sus raíces, han conservado intacta la colocación y el arreglo de las piedras de construcción. Es bastante interesante ver cómo las raíces de árboles han logrado preservar arreglos arquitectónicos desde el tiempo de los mayas. En la zona de Las Sepulturas (Hendon, Fash y Aguilar Palma 1990: 132; Láminas 10 y 11) hay una muestra clara del milagro que hicieron las raíces de un árbol. Allí logró conservarse un arreglo especial de piedras labradas. 


\section{Observaciones en Copán}

Una vez hechas las abras entre las ramas de los árboles, para poder observar las salidas o puestas del sol y estando la Profesora María Cristina presente para estas observaciones, se iniciaba con las mismas. Los muchachos de limpieza regresaban a sus puestos habituales de trabajo y nosotros hacíamos arreglos con el Representante del Instituto para poder entrar a las ruinas, por lo general, a las 5:00 am. Para la tarde se hacía arreglos para salir ya un poco tarde, después de las 6:00 pm. Los vigilantes de las Ruinas quedaban sabidos, desde el día anterior, de los arreglos y a las cinco de la mañana entrábamos a las ruinas. Muchas veces se nos unía el esposo de la Profesora y, de vez en cuando, algunos otros miembros del Observatorio. La Profesora nos asignaba el punto desde donde observaríamos la salida del sol. Nos poníamos en frente de un monumento, viendo hacia el este y con una cámara lista para tomar fotografías.

A pesar de que ahora tenemos una fecha más temprana de la que utilizamos inicialmente, la Estela $\mathrm{C}$ del Patio del Sol tiene el privilegio de haber sido la primera colocada en este patio, en el año 711 d.C. La Estela F fue la segunda erigida en este patio en el año 726 d.C. y, viendo de la Estela $C$ hacia la F, durante los equinoccios (generalmente los 21 de marzo y de septiembre), se ve salir el sol, directamente detrás de la Estela $F$ (Figura 1). De esta foto queda claro, no hay ninguna duda, de que el sol, al salir del horizonte, está alineado con estas dos estelas. Queda claro que no hay ninguna farsa ni mentira. Los equinoccios son los momentos cuando el día y la noche tienen igual duración. En ese día, el sol marca el punto intermedio, geográfico y temporal, entre los solsticios. 


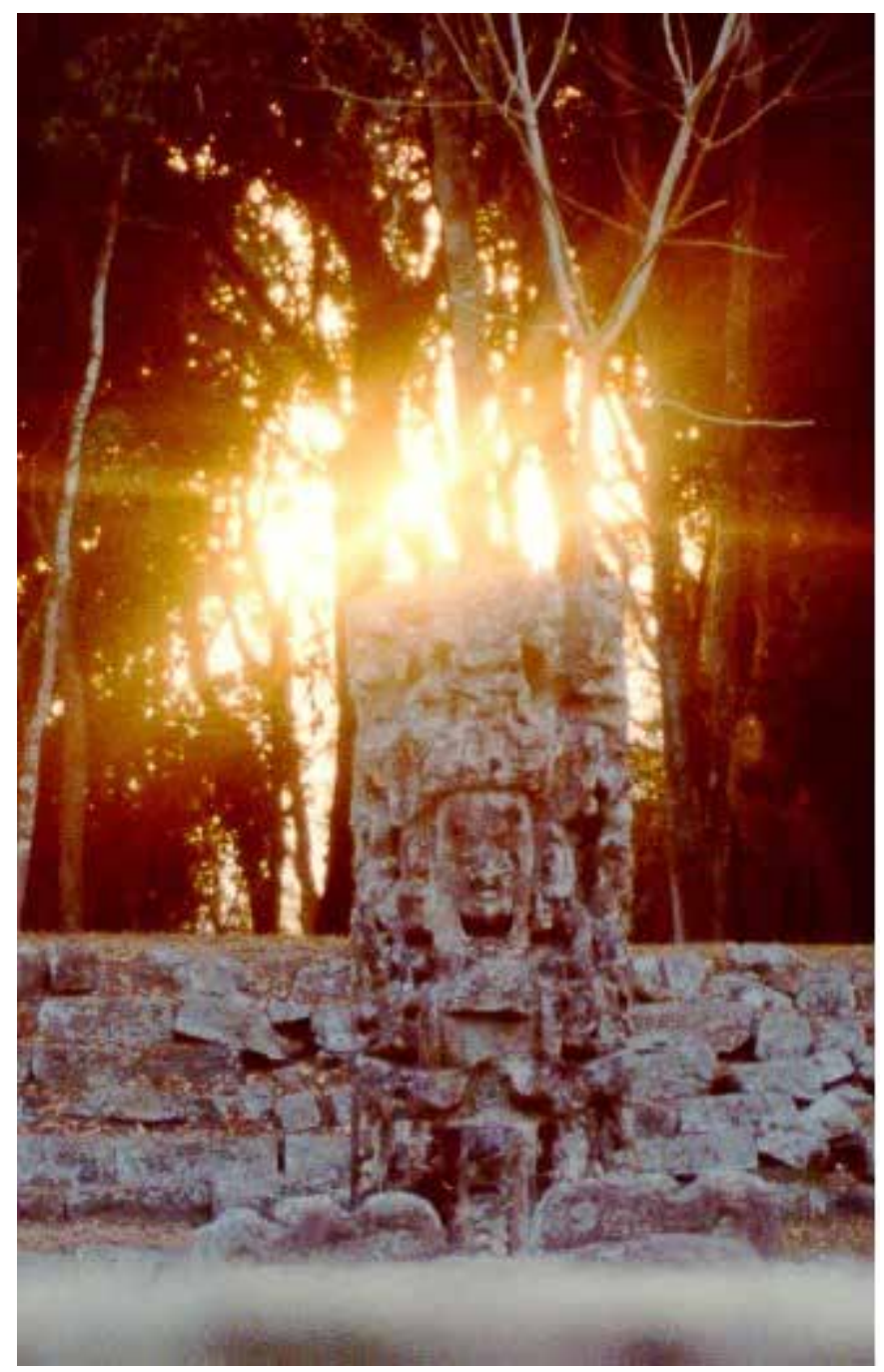

Figura 1. Salida del sol, el día del equinoccio de primavera, viendo de la Estela C a la F.

Otro ejemplo es la salida del sol en los pasos del sol por el cenit (por lo general el 30 de abril y el 12 de agosto, en Copán Ruinas). Como prueba utilizamos aquí un solo monumento. Se trata de la sección de la gradería noroeste, al norte del Patio del Sol. La guía en este caso es la dirección de las graderías (Figura 2). Aquí no hay inventos. La dirección de las graderías está orientada hacia la salida del sol. Éste es un aspecto muy importante porque nos explica la razón por la cual estas graderías están orientadas en esa dirección. Primera vez que se propone esta explicación en cuanto a las diferencias de las direcciones de este extremo 
norte del patio. Comparando este patio con otro similar en Quiriguá, aquel tiene un final normal, una línea recta. Como veremos con el lado noroeste, los dos están señalando la salida del sol en un día de evento especial del sol.

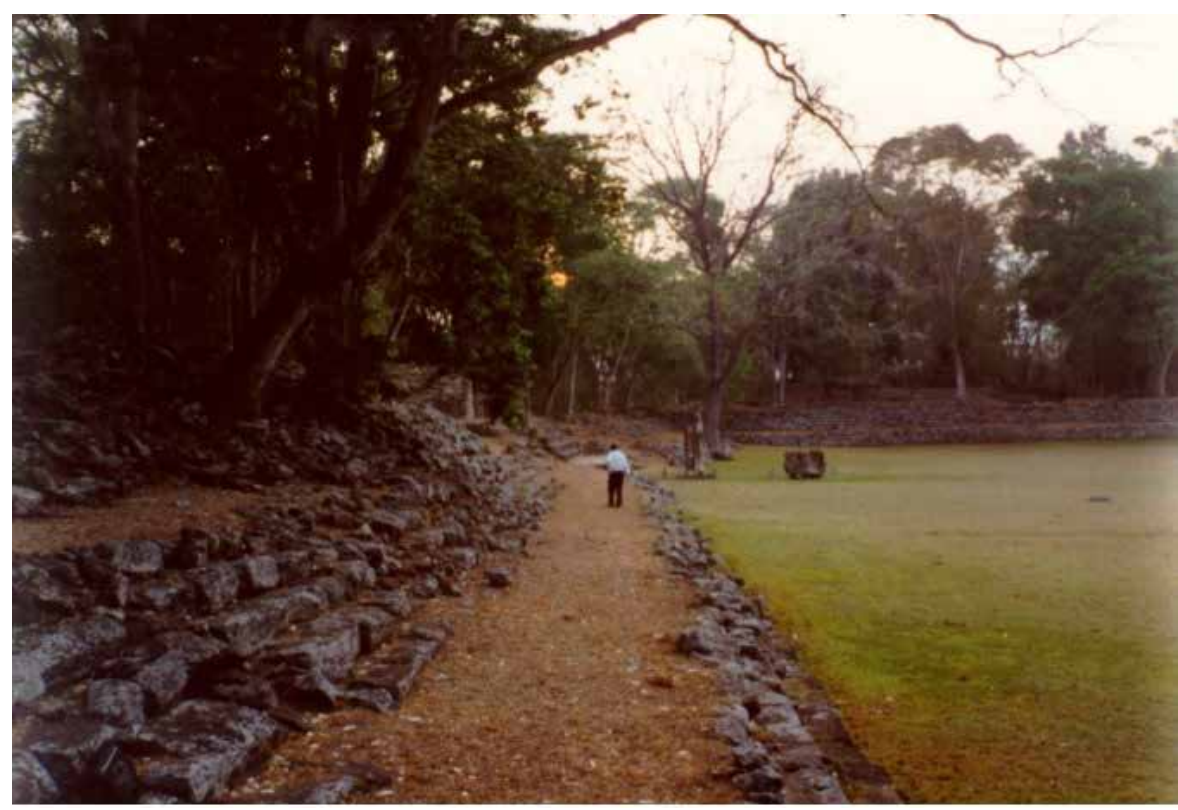

Figura 2. Salida del sol, el día del paso del sol por el cenit, a lo largo de la Gradería NO del Patio del Sol.

Para el solsticio de verano (en general el 21 de junio), tenemos la salida del sol, viendo de la Estela 4 a la Estela F (Figura 3). Éste es el día más largo y la noche más corta del año y el día cuando los monumentos arrojan la sombra más larga hacia el sur. La sombra no es muy larga porque el sol solamente se aleja unos $809^{\circ}$ hacia el norte de Copán. 


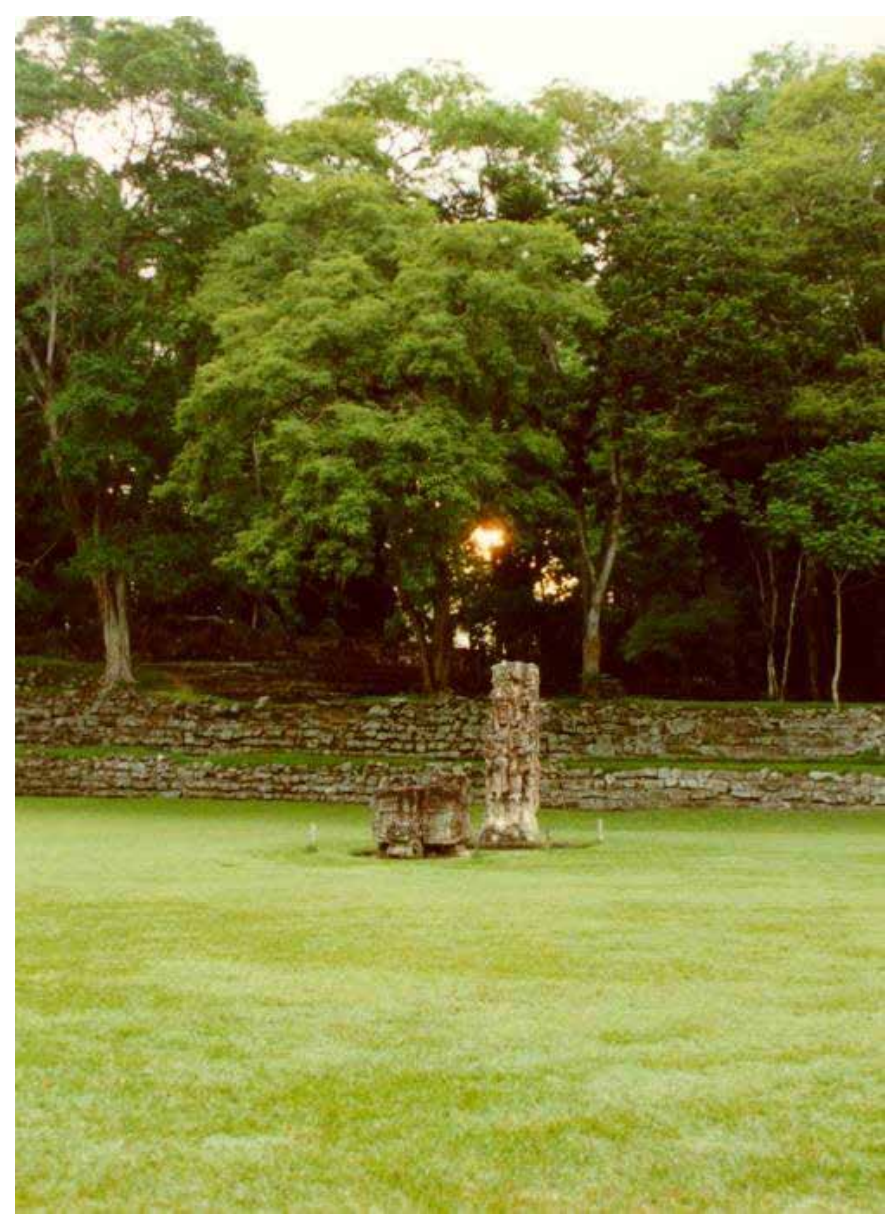

Figura 3. Vista de la salida del sol en el solsticio de verano, viendo de la Estela 4 a la F.

Por fin y en cuanto a salidas, tenemos el alineamiento entre la Estela $E$ y la esquina NE de la Estructura 4, el día del solsticio de invierno, alrededor del 21 de diciembre (Figura 4). Éste es el día cuando la sombra de los monumentos se proyecta lo más hacia el norte y es la sombra más larga del año. Es la más larga porque el sol anda casi $48^{\circ}$ hacia el sur y, entre más lejos está la fuente de luz, más larga es la sombra. 


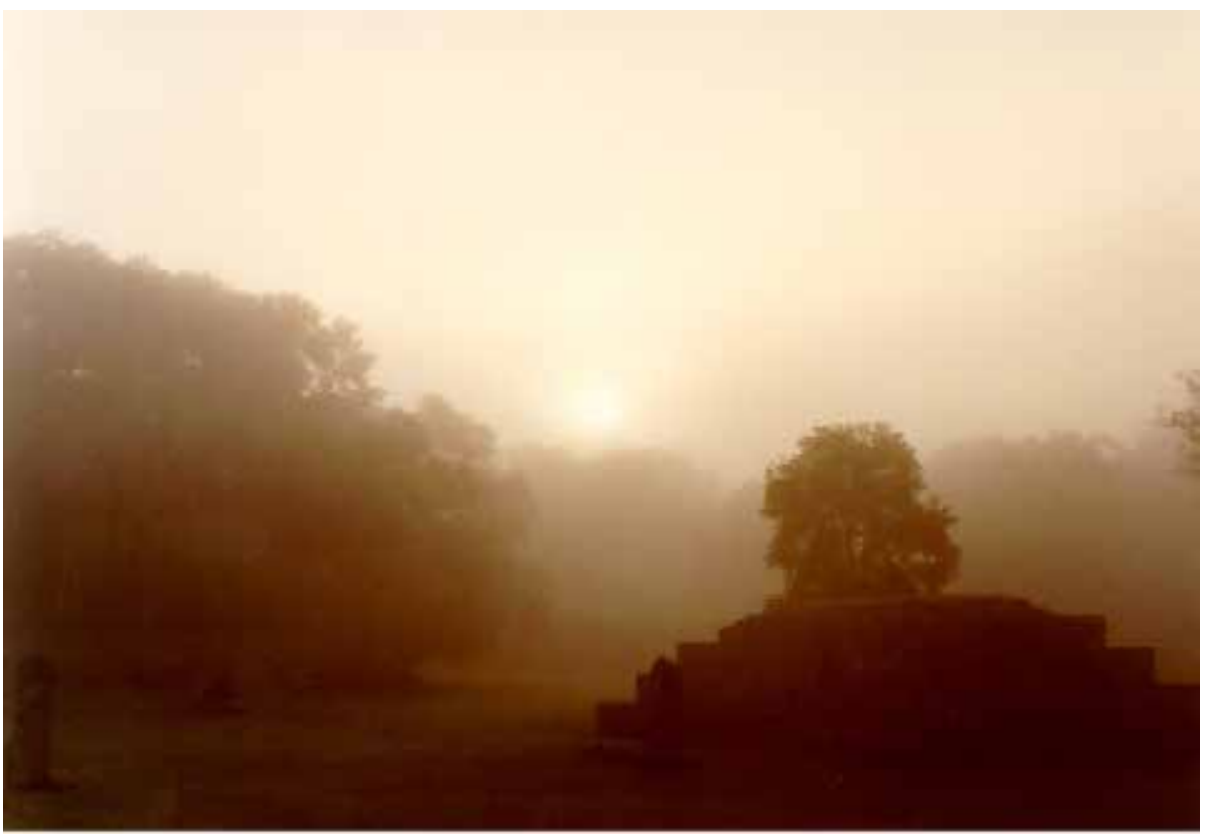

Figura 4. Salida del sol durante el solsticio de invierno, viendo de la Estela E hacia la esquina NE de la Estructura 4.

Ahora, con respecto a las puestas, tenemos que, para los equinoccios, basta con darse vuelta, en el mismo punto de observación de la salida del sol, y se verá la puesta, de manera inversa.

Con respecto al paso del sol por el cenit, para la puesta del sol, tenemos la vista a lo largo de las graderías de la Estructura 223. Allí se verá la puesta del sol, en la dirección de esas graderías (Figura 5). La dirección de esta estructura se diferencia de las otras por su inclinación hacia el noroeste. Es una inclinación que, los arqueólogos, quienes la excavaron, no pudieron explicar (Cheek y Spink 1986: 82-83). Para ellos, la Estructura 223 está a $15^{\circ}$ al sur del este, ángulo extraño, comparado con el grupo entero. Se considera que nosotros hemos encontrado la razón para esta inclinación: siguiendo los pasos del sol por el cenit. 


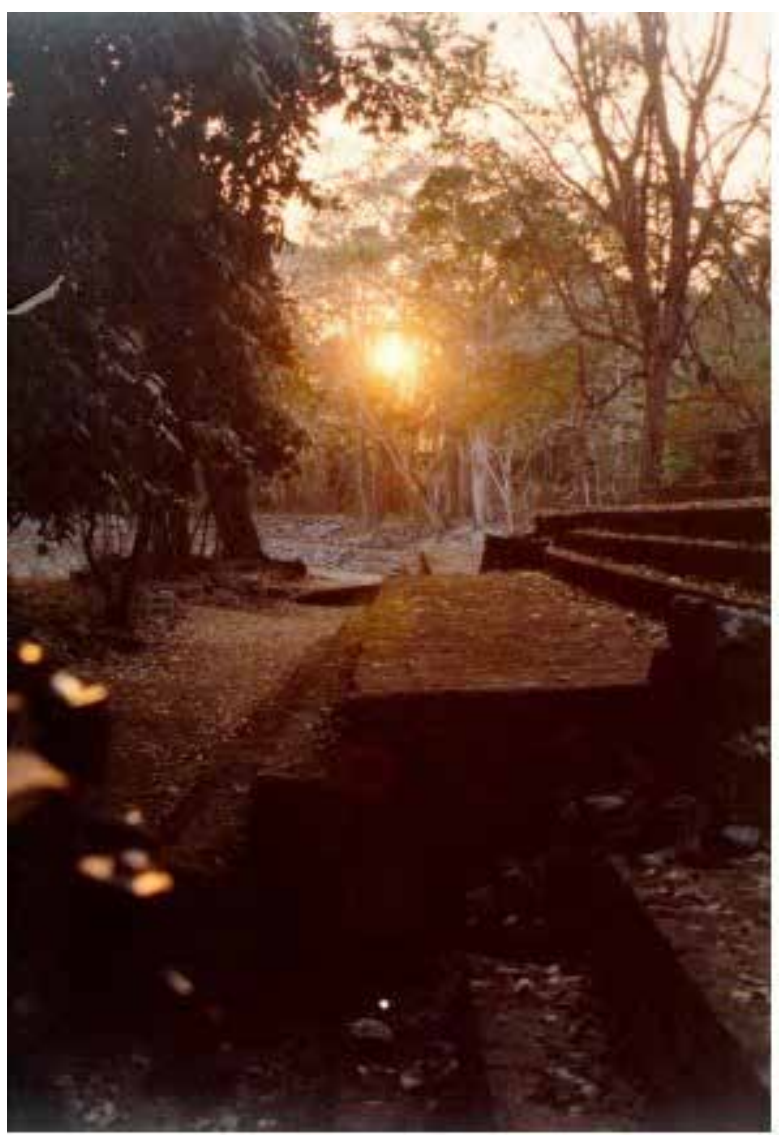

Figura 5. Puesta del sol el día del paso del sol por el cenit, viendo a lo largo de las graderías de la Estructura 223.

Para la puesta del sol en el solsticio de verano, tenemos la vista de la Estela $\mathrm{H}$ a la Estela 4 (Figura 6). Es éste el día más largo del año y la noche más corta. Es el día cuando la sombra es la más corta que arrojan los monumentos hacia el sur, pues el sol no está muy bajo, por estar a solamente unos $9^{\circ}$ al norte de Copán. En esta vista está claro que el sol se pone detrás de la Estela 4. 


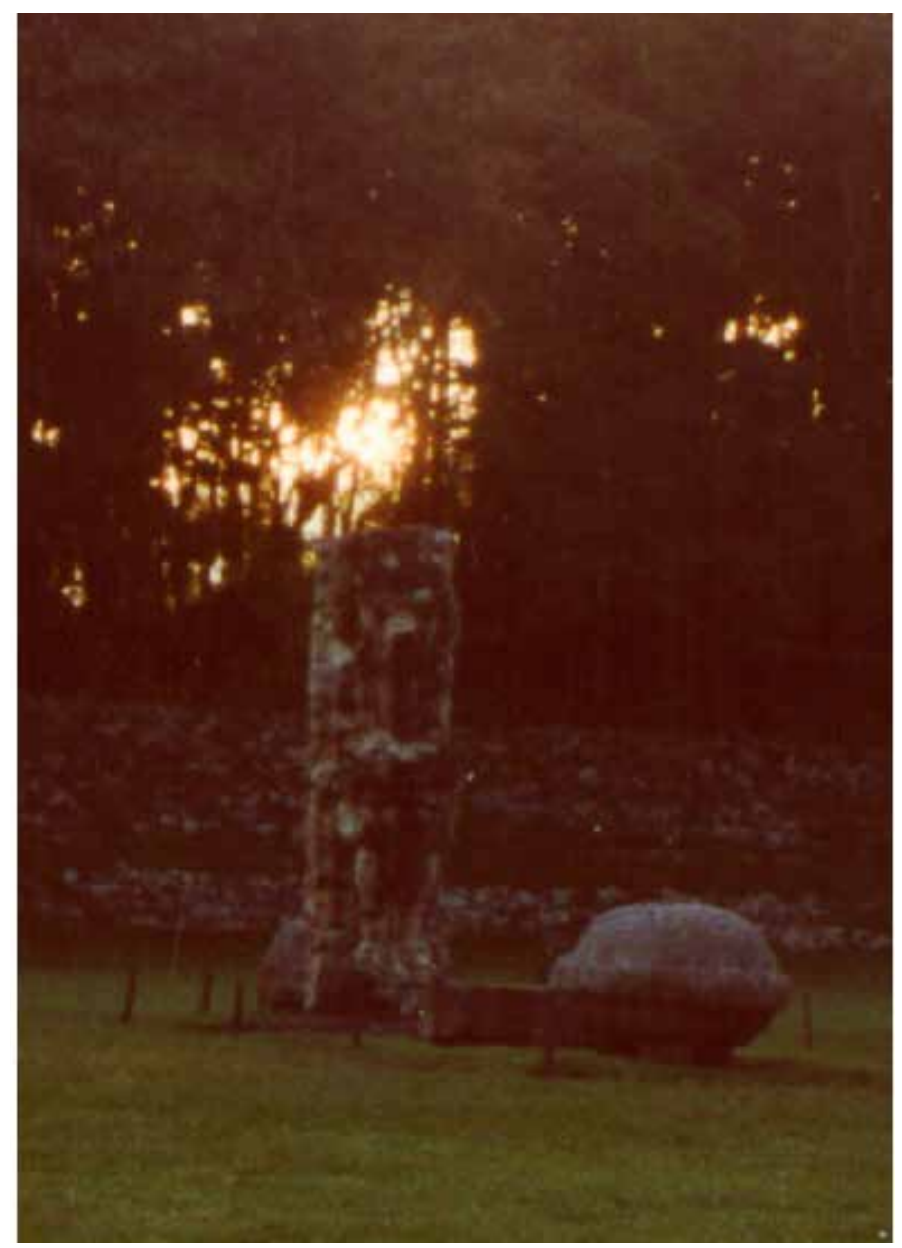

Figura 6. Puesta del sol, durante el solsticio de verano, viendo de la Estela $\mathrm{H}$ a la Estela 4.

\section{Resultados Diseminados}

Con la ayuda especialmente del Ing. Raúl Welchez en Copán Ruinas, se logró comenzar a dar a conocer los resultados de nuestros hallazgos. En cada visita que se hacía a Copán Ruinas para hacer observaciones, se ofrecía una charla sobre el tema de la visita. Una vez hablábamos de los equinoccios, otra sobre los pasos del sol por el cenit, otra vez hablábamos del solsticio de verano y, por fin, sobre el solsticio de invierno. 
Las charlas eran por lo general en el Hotel Marina, donde el Ing. Welchez nos proporcionaba el lugar, una computadora y nos ayudaba a hacerle publicidad al evento. La publicidad la hacía por medio de internet, enviando mensajes a varias instituciones e individuos locales. De manera que, en cada charla, había una participación aceptable, alrededor de unas veinte personas por charla. En cada charla se explicaba el evento solar, sus especialidades, la fecha, los lugares que se habían seleccionado para su observación. Al mismo tiempo se invitaba a la gente para que nos acompañara temprano por la mañana y por la tarde a observar la salida y la puesta del sol y también las sombras de los monumentos al medio día. Muchas veces se registraba la participación de grupos fuera de Copán y de grupos de estudiantes. Era interesante ver la participación de estas personas. Por las preguntas que hacían, se notaba el interés y el poco conocimiento que tenían sobre los temas.

Después de esos eventos en Copán Ruinas, regresábamos a Tegucigalpa, para continuar con la divulgación de esos eventos. En primer lugar, hacíamos divulgación entre nuestras clases de "Introducción a la Arqueoastronomía". En ellas se explicaba a los estudiantes la metodología que seguíamos y luego los resultados. Se hacía hincapié para que los estudiantes vieran las oportunidades que esto nos daba para conocer más sobre nuestras raíces.

También haciamos todo el esfuerzo posible para diseminar estos resultados, publicando notas en los periódicos y por medio de entrevistas con distintos medios de comunicación del país. Los medios de comunicación eran muy instrumentales en dar a conocer los resultados. Nos hacían entrevistas, las cuales resultaban muy interesantes y ellos eran muy respetuosos de los datos y publicaban los datos exactos.

\section{Conservación de los Monumentos}

De acuerdo con distintas definiciones, el Instituto Hondureño de Antropología e Historia resume bien la idea de monumento en su Ley para la Protección del Patrimonio Cultural, diciendo que son aquellos bienes inmuebles, ubicados en el mismo predio público o privado, de la época precolombina, colonial y republicana que, por su arquitectura o ingeniería, sean de interés antropológico e histórico.

Uno de los objetivos del IHAH es conservar estos bienes. El Departamento de Arqueoastronomía y Astronomía Cultural tiene como uno de sus objetivos conservar estos bienes también. De acuerdo con definición del IHAH, conservación (preservación) es el conjunto de principios y técnicas encaminadas a la protección de los Bienes Arqueológicos contra los agentes que puedan destruirlos. 
Uno de los aspectos primordiales para conservar algo es conociéndolo y la tarea de llegar a conocer nuestros bienes culturales la realizamos haciendo las observaciones. Por medio de ese esfuerzo, llegamos a conocer sobre lo que estamos hablando. En primer lugar, conociendo sobre los movimientos solares y de otros astros. Así llegamos a conocer los días exactos en que suceden esos momentos importantes del sol. Decimos del sol, en este caso, porque es el único astro que hemos estado estudiando.

Por medio de nuestras observaciones, llegamos a conocer estos hechos y por medio de nuestros escritos y conferencias damos a conocer nuestros conocimientos a los demás. Los conocimientos que transmitimos son los referentes a los movimientos aparentes de los astros, especialmente el sol. Se dice "aparentes" porque no es el sol el que se mueve, sino nosotros, junto con la tierra, en sus movimientos de rotación y de traslación.

Rotación es el movimiento de la tierra alrededor de su eje en un día, del cual nosotros tenemos el día y la noche. Entonces, nosotros vemos salir el sol, todos los días por el este. Pero no es el sol el que sale. Somos nosotros los que le aparecemos al sol. Traslación es el movimiento de la tierra alrededor del sol, que realiza en un año. El sol no sale ni se pone a la misma hora ni en el mismo lugar todos los días. Cada día sale a una hora distinta y en un lugar distinto. El día del solsticio de invierno es el día más corto del año y la noche más larga del año y el sol sale y se pone en el punto más al sur. Solsticio viene de dos palabras latinas: sol que quiere decir sol y stat que quiere decir se detiene. A partir del solsticio de invierno, el sol va saliendo más al norte y los días comienzan a ser más largos y las noches más cortas.

El día del equinoccio de primavera es cuando el día y la noche son iguales. La palabra se compone de dos vocablos latinos: equi que quiere decir igual y noccio que quiere decir noche. Entonces, la palabra equinoccio quiere decir igual noche (al día).

El paso del sol por el cenit es algo que ocurre únicamente en la franja tropical: 23.5 grados al norte y 23.5 grados al sur del Ecuador Terrestre. Este hecho no ocurre, ni en la misma fecha ni en todo el mundo, como los otros días (solsticios y equinoccios). Dentro de esa franja, el día del paso del sol por el cenit depende de la posición norte-sur en que esté el lugar. Por ejemplo, aquí en Honduras, el primer paso del sol por el cenit sucede entre fines de abril y principios de mayo, comenzando en Choluteca y terminando en Islas de la Bahía. El segundo paso del sol por el cenit sucede al contrario, comienza en Islas de la Bahía y termina en Choluteca. 
Es el día cuando los rayos del sol caen directamente, en aquellos lugares donde está sucediendo ese evento. Por esa razón son días de mayor calor. Pero no tan exagerado, como nos hacen creer algunos de los medios de comunicación.

El solsticio de verano es el día cuando el sol llega al punto más al norte, en su recorrido de sur a norte. Ese día por lo general sucede el 21 de junio, de cada año. Es el día cuando el sol está en el Trópico de Cáncer al norte y es el día más largo de todo el año y la noche más corta de todo el año. Hay ciertas regularidades los días de los solsticios. Por ejemplo, la dirección en que sale el sol en un solsticio es la misma dirección en que se pone en el otro solsticio. Igualmente, la dirección en que se pone en un solsticio es la dirección en que sale en el otro solsticio.

Luego viene el segundo paso del sol por el cenit, que generalmente sucede el 12 de agosto. Tiene las mismas características que el primer paso del sol por el cenit, nada más que los rayos del sol no son tan ardientes. Es momento de cosecha y de regocijo entre la gente porque vienen saliendo las cosechas y van terminando los días de mucha escasez de alimentos.

Se continúa con el equinoccio de otoño, que tiene las mismas características que el equinoccio de primavera.

Por fin llegamos al solsticio de invierno, por lo general el 21 de diciembre. En el hemisferio norte es un momento de frío, lluvias y quizá hasta huracanes. Es el día más corto y la noche más larga de todo el año.

Debe recordarse que, a partir del Ecuador, la Tierra está dividida en dos hemisferios: el hemisferio norte y el hemisferio sur. Las estaciones del año en estos dos hemisferios se suceden opuestamente. Por ejemplo, cuando en el hemisferio norte es el solsticio de verano, en el hemisferio sur es el solsticio de invierno. Cuando en el norte es el equinoccio de primavera en el sur es el equinoccio de otoño. Cuando en el hemisferio norte es el solsticio de verano, en el sur es el solsticio de invierno. Cuando en el hemisferio norte es el equinoccio de otoño, en el sur es el equinoccio de primavera.

\section{Lecciones para la Identidad Nacional}

Dejando por fuera muchas otras fuentes de identidad nacional, aquí podemos encontrar varias lecciones de identidad.

Puede decirse, en general, que estos distintos movimientos del sol nos dejan distintas impresiones. Una de ellas, muy palpable, es el hecho de una ines- 
tabilidad mental bien clara. Ya que el sol se nos presenta en distintas variedades y situaciones: unas veces al sur, otras veces al norte, otras veces sobre nuestras cabezas, unas veces con la sombra al norte, otras veces al sur y algunos días sin sombra; todo esto nos hace bien inestables: "que si", "que no", "quizá", "tal vez", "es posible", "puede ser", etc. Muchas veces, nosotros no somos "ni chicha ni limonada". Esto hablando de manera general.

En forma particular y tomando en cuenta cada evento solar, comenzando con el equinoccio de primavera, puede decirse que éste es el momento cuando para nosotros comienza el verano. Hay que rozar los terrenos, quemar antes de las lluvias y por fin sembrar. Durante la espera de la siguiente cosecha, es por lo general un momento de gran expectativa porque no hay alimentos. Mucha gente sufre hambre. A partir del equinoccio de otoño, comienzan las lluvias, los huracanes y son momentos de mucha preocupación.

Todos esos estados de mente, trabajan en nuestro ser y moldean nuestro modo de pensar y nos llevan a actuar en cierta forma: a la indecisión, a la fuerza de voluntad de aguantar hambre, a la fuerza de voluntad de aguantar las embestidas de la naturaleza.

\section{Conclusiones}

Ésta es una de las primeras pruebas de arqueoastronomía realizada por hondureños. A pesar de ser un campo nuevo, tuvieron el valor de lanzarse a esta aventura, pero con resultados muy positivos. En primer lugar, hicieron cálculos en Tegucigalpa sobre los posibles alineamientos en Copán. Tomaron mapas de las ruinas e hicieron cálculos de los posibles alineamientos de ciertos monumentos con las salidas o puestas del sol.

Luego se hizo todos los arreglos en Copán para poder hacer las observaciones, las cuales se realizaron con mucho éxito. Primero haciendo todos los arreglos de limpieza y luego realizando las observaciones. Por medio de éstas se comprobó que realmente es sol salía y se ponía por los puntos previstos. Los alineamientos pudieron comprobarse viendo de un monumento a otro y luego el sol o a lo largo de una gradería.

Pudo así comprobarse que los gobernantes mayas habían seleccionado esos días para hacer esas observaciones con grupos de su gente, desde puntos especiales. 
Ésta es una pequeña muestra de lo que se puede hacer en Copán. Debe recordarse que se ha trabajado solamente sobre el sol y solamente sobre una sección del sitio de Copán. El trabajo debe extenderse a otras zonas de Copán, a otros sitios mayas y a otros astros. Así se estará comprobando que estas cosas sucedían no sólo en Copán sino también en otros sitios mayas.

\section{Agradecimientos}

Se agradece la colaboración y la participación de la Profesora María Cristina Pineda de Carías y de su esposo, Marcos Carías. También se toma en cuenta la participación de varios de los miembros de la Facultad de Ciencias Espaciales. Mucho se agradece la colaboración de Ricardo Agurcia, quien siempre estuvo pendiente de las investigaciones y de sus buenos resultados. Se está muy agradecido con Seiichi Nakamura por su disposición positiva a colaborar con el proyecto. También se agradece al Señor Raúl Welchez por su continua y decidida colaboración a lo largo de todo el proyecto. Se agradece a algunos de los guías de Copán por su participación en las observaciones y en las charlas en Copán Ruinas. Por fin se agradece al Instituto Hondureño de Antropología e Historia por su decidida y positiva colaboración en todos los aspectos del proyecto.

\section{Bibliografía}

- Aveni, Anthony F. 1972 Astronomical Tables Intended for Use in Astro-archaeological Studies. American Antiquity 37 (4, Oct.): 531-540.

- Aveni, Anthony F. 1980 Conceptos de Astronomía Posicional Empleados en la Arquitectura Mesoamericana Antigua. En Astronomía en la América Antigua (Anthony F. Aveni, compilador): 23-42. Siglo XXI, México.

- Aveni, Anthony F. La Arqueoastronomía y la conservación del patrimonio cultural hondureño

- Aveni, Anthony F. 1980a Skywatchers of Ancient Mexico. University of Texas Press, Austin.

- Aveni, Anthony F. 1997 Stairways to the Stars: Skywatching in Three Great Ancient Cultures. John Wiley \& Sons, Inc., New York.

- Aveni, Anthony F. y Horst Hartung, 1976 Investigación Preliminar de las Orientaciones Astronómicas de Copán. Yaxkin I (3): 8-13. Instituto Hondureño de Antropología e Historia, Tegucigalpa. 
- Aveni, Anthony F. y Horst Hartung, 2005 Observadores del Cielo en el México Antiguo. Fondo de Cultura Económica, México.

- Broda, Johanna,1982 Arqueoastronomía y Desarrollo de las Ciencias en el México Prehispánico. En Simposio de Historia de la Astronomía en México (Marco Arturo Moreno Corral, editor): 69-117. Instituto de Astronomía, Observatorio Astronómico Nacional, Universidad Nacional Autónoma de México.

- Carlson, John B., David S. P. Dearborn, Stephen C. McCluskey y Clive L. N. Ruggles, 1999 Astronomy in Culture. Archaeoastronomy: The Journal of Astronomy in Culture XIV (1): 3-21.

- Cheek, Charles D. y Mary L. Spink. 1986 Excavaciones en el Grupo 3, Estructura 223 (Operación VII). En Excavaciones en el Área Urbana de Copán, Tomo I, William T. Sanders, Director. Proyecto Arqueológico Copán, Segunda Fase. Instituto Hondureño de Antropología e Historia, Tegucigalpa.

- Hawkins, Gerald,1965 Stonehenge Decoded. Doubleday, Nueva York.

- Hendon, Julia A., William L. Fash y Eloísa Aguilar Palma, 1990 Excavaciones en 9N-8, Conjunto del Patio B. En Excavaciones en el Ärea Urbana de Copán, Tomo II, William T. Sanders, Director. Secretaría de Cultura y Turismo, Instituto Hondureño de Antropología e Historia. Tegucigalpa, 1990.

- Hohmann, Hasso y Annegrete Vogrin,1982 Die Architektur von Copan (Honduras), 2 Vols. Akademische Druck-und Verlagsanstalt, Graz.

- Iwaniszewski, Stanislaw.1982 Mitología y Arqueoastronomía: La Interpretación Astronómica de los Mitos. En Simposio de Historia de la Astronomía en México (Marco Arturo Moreno Corral, editor): 119-149. Instituto de Astronomía, Observatorio Astronómico Nacional, Universidad Nacional Autónoma de México.

- Maupomé, Lucrecia,1982 Reseña de las Evidencias de la Actividad Astronómica en la América Antigua. En Simposio de Historia de la Astronomía en México (Marco Arturo Moreno Corral, editor): 9-68. Instituto de Astronomía, Observatorio Astronómico Nacional, Universidad Nacional Autónoma de México.

- Morley, Sylvanus G., 1920 The Inscriptions at Copan. Carnegie Institution of Washington. Publication 219. Washington, D.C.

- Pineda de Carías, María Cristina, Vito Véliz y Ricardo Agurcia Fasquelle,2002 Acerca de las Observaciones del Sol Realizadas en la Gran Plaza del Par- 
que Arqueológico de Copán Ruinas, Honduras. Yaxkin XXI: 15-44. Órgano de Divulgación del Instituto Hondureño de Antropología e Historia. Publicación Anual, Tegucigalpa.

- Pineda de Carías, María Cristina, Vito Véliz y Ricardo Agurcia Fasquelle, 2002a El Grande y Complejo Plan de 18 Conejo para la Construcción de la Plaza del Sol del Parque Arqueológico de Copán, Honduras. En Revista IHAH : Cincuenta Años con el Patrimonio Cultural de la Nación, Edición Conmemorativa del Instituto Hondureño de Antropología e Historia (1952-2002). Tegucigalpa. 